\title{
Feasibility of radical hysterectomy in women with FIGO stage IIB cervical cancer: an observation study of 10-year experience in a tertiary center
}

This article was published in the following Dove Press journal: OncoTargets and Therapy

\author{
Lei Yuan ${ }^{1, *}$ \\ Jiaqi Guo ${ }^{1, *}$ \\ Xiaochun Zhang ${ }^{2}$ \\ Mo Chen' \\ Congjian $\mathrm{Xu}{ }^{\prime}$ \\ Liangqing Yao' \\ 'Department of Gynecology, \\ Obstetrics and Gynecology Hospital, \\ Fudan University, Shanghai, People's \\ Republic of China; ${ }^{2}$ Department of \\ Obstetrics and Gynecology, Fenyi \\ People's Hospital, Jiangxi, People's \\ Republic of China \\ *These authors contributed equally \\ to this work
}

Correspondence: Liangqing Yao Department of Gynecology, Obstetrics and Gynecology Hospital, Fudan University, 419 Fangxie Road, Shanghai 2000 I I, People's Republic of China

Tel +86 I39 I664 4444

Fax +86 2I 63450944

Email yaoliangqing@163.com
Purpose: Although definitive chemoradiotherapy is considered as a standard of care for FIGO stage IIB cervical cancer in many countries, the role of surgery remains controversial. We aimed to evaluate the feasibility and outcomes of patients with FIGO stage IIB cervical cancer who received radical surgery in China.

Patients and methods: A total of 74 women with FIGO stage IIB cervical cancer were treated with radical hysterectomy, with or without adjuvant radio/chemoradiotherapy, at the Obstetrics and Gynecology Hospital of Fudan University between 2004 and 2015. Medical charts and clinical data were retrospectively reviewed. The Kaplan-Meier method and Cox regression models were used for survival analyses. In addition, prognostic nomograms predicting overall survival (OS) and progression-free survival (PFS) were constructed.

Results: Pathological parametrial involvement (PMI) was only identified in $28.3 \%$ (21/74) of all patients and $47.3 \%(9 / 19)$ of patients without neoadjuvant treatment. Major surgical complications, including bladder fistula, intestinal obstruction and ureteral injury, were found in $6.8 \%(5 / 74)$ of patients. Although the use of imaging technologies including magnetic resonance imaging (MRI)/positron emission tomography-computed tomography (PET-CT) has increased after 2010 compared to that prior to 2010, the accuracy of MRI/PET-CT in detecting pathological PMI was lower than that of physical examination under anesthesia $(P<0.05)$. Neoadjuvant treatment was the only risk factor affecting the accuracy of pre- and postoperative accordance of PMI (OR: 3.283 [95\% CI: 1.363-7.908], $P=0.008$ ). The 2- and 5-year OS rates were 84.1\% and $68.9 \%$, respectively, while the 2 - and 5 -year cumulative recurrence rates were $26.9 \%$ and $39.9 \%$, respectively. Cox regression analyses indicated that pre- and postoperative accordance of PMI, common iliac lymph node metastasis and major surgical complications were significant prognostic factors for both OS and PFS.

Conclusion: Radical hysterectomy might be a feasible alternative for FIGO stage IIB cervical cancer. As pre- and postoperative accordance of PMI is relatively low, strategies to appropriately select patients who will benefit from surgery via pretreatment evaluation need to be further investigated.

Keywords: locally advanced cervical cancer, parametrial involvement, radical hysterectomy, surgery

\section{Introduction}

Cervical cancer is the third most common cancer in women worldwide, with an annual global incidence of 528,000 and an annual death rate of 275,000 in 2012. ${ }^{1}$ Approximately $85 \%$ of newly diagnosed cases occur in developing countries, where cervical cancer is a leading cause of cancer death in women., ${ }^{2,3}$ This phenomenon is particularly 
true in China, where $>135,000$ cases of cervical cancer are diagnosed every year. In addition, epidemiological surveys have shown that patients with cervical cancer have tended to be younger in China in the past decade, and patients in rural areas usually suffered from more advanced stages and higher mortality. ${ }^{4}$ Therefore, the situation in China is rather serious with a large patient base and a relatively high prevalence of advanced-stage disease.

Surgery, definitive radiation and surgery combined with adjuvant radiation/chemoradiation therapy are currently the three predominant treatment arms for cervical cancer. Decades have witnessed changes in treatment recommendations for stages I-II cervical cancer in light of a number of randomized trials conducted over various time periods. For example, studies of both surgery vs radiation for early stage cervical cancer and radiation alone vs chemoradiation have shaped current treatment guidelines. ${ }^{5-7}$ However, controversy remains regarding the treatment modalities for patients with locally advanced cervical cancer (LACC), especially patients with FIGO stage IIB disease.

As a clinical staging system that largely depends on pretreatment physical examination by surgeons, the sensitivity and specificity of FIGO staging are rather disappointing. Data have shown that the incidence of pathologically proven parametrial involvement (PMI) among patients with FIGO stage IIB disease ranges between $21.5 \%$ and $55 \% .{ }^{8,9}$ That is, more than half of the patients with FIGO stage IIB disease clinically have "pseudo" stage IIB disease, which can be treated by surgery but not definitive radiation. Moreover, a desire for hormone preservation among young patients should also be carefully considered when making a plan of definitive radiotherapy. ${ }^{10}$

In addition, surgery facilitates the accurate assessment of histopathological findings. Several retrospective studies have demonstrated a significant rate of upstaging, mainly determined by nodal or peritoneal spread, as a confirmation of more extensive disease in a substantial proportion of patients when comparing the results of standard clinical staging with pathological features after surgery. ${ }^{11,12}$ Thus, an individualized extended field of radiation could be implemented to patients according to pathological findings and might be beneficial for their overall survival (OS). ${ }^{13}$

Finally, in developing countries, limited access to radiotherapy, as well as disparities in standard treatment due to low income and lack of medicaid, ${ }^{14}$ may also contribute to the preference of surgery over radiation.

Therefore, the aims of this study were to evaluate the feasibility of radical hysterectomy in women with FIGO stage IIB cervical cancer and determine their prognostic factors in a setting of a long-term follow-up study in a Chinese tertiary center.

\section{Patients and methods}

\section{Patient selection}

A total of 74 patients with FIGO stage IIB cervical cancer who had been admitted and surgically treated at the Obstetrics and Gynecology Hospital of Fudan University from January 2004 to December 2015 were enrolled in this study. All patients received radical hysterectomy plus systematic pelvic lymphadenectomy with or without adjuvant postoperative radio/chemoradiotherapy according to the Sedlis criteria. Neoadjuvant treatment, including neoadjuvant chemotherapy or chemoradiotherapy, was also provided or not according to patient preference and surgeon expertise. Regular surveillance including physical examination, cervical/vaginal cytology and imaging was carried out every 3 months for 2 years post operation, every 6 months for 3 years, and every year after 5 years. Overall, 71 patients were followed up with a median follow-up duration of 36 months (range: 7-143 months).

Medical records were reviewed, and demographic data, clinicopathological characteristics and outcome data, including intraoperative blood loss, perioperative major complications and survival, were analyzed. Major complications included ureteral injury and obstruction with a need for a double-J tube insertion, bladder fistula and intestinal obstruction. For follow-up, OS was calculated from the date of radical hysterectomy to the date of death or last follow-up, and progression-free survival (PFS) was calculated from the date of radical hysterectomy to the date of cancer recurrence or last follow-up. All procedures in this study were in accordance with the 1964 Declaration of Helsinki, and the study was approved by the institutional ethics review board of the Obstetrics and Gynecology Hospital of Fudan University. All patients provided written informed consent for review of their medical records.

\section{Statistics}

Descriptive statistics were used to characterize the study cohort. The comparison of distributions of continuous variables between or among two or more groups was performed using the Wilcoxon and Kruskal-Wallis tests, respectively. Survival curves were depicted using the Kaplan-Meier method and compared using the log-rank test. The significance level for abovementioned analyses was 0.05. A forward conditional (Wald) Cox proportional hazard model was used 
for multivariate analyses. A $P$-value of $<0.10$ was considered to be significant for entry, and $P<0.05$ was required for retention in the model.

In addition, a prognostic nomogram was formulated based on the results of multivariate analysis and by using the $\mathrm{rms}^{15}$ package in R version 2.14.1 (http://www.r-project.org/). The performance of the nomogram was measured by the concordance index (C-index). All other computations were done with SPSS 16.0 (SPSS Inc., Chicago, IL, USA).

\section{Results}

\section{Clinical and pathological features of patients with FIGO stage IIB cervical cancer}

A total of 74 patients with an average age of 50.0 \pm 8.7 years were identified with chief complaints of irregular vaginal bleeding (38 cases, 51.4\%), contact vaginal bleeding (22 cases, 29.7\%), vaginal discharge (nine cases, 12.2\%) or by occasional findings on physical examination (five cases, 6.7\%). All patients underwent radical hysterectomy and pelvic lymphadenectomy via laparotomy or laparoscopy. The clinical and pathological characteristics are summarized in Table 1.

Among all patients with clinical stage IIB disease, only 21 had pathologically confirmed PMI after surgery. Thus, the pre- and postoperative accordance rate of PMI was as low as $28.4 \%(21 / 74)$. For a subset of patients without neoadjuvant treatment, the accordance rate of PMI was increased to $47.3 \%(9 / 19)$. For patients with pelvic lymph node metastasis, $48.4 \%$ (15/31) had positive common iliac nodes. In addition, postoperative adjuvant chemotherapy/ radiochemotherapy was administered in $16.2 \%(12 / 74)$ and $74.3 \%(55 / 74)$ patients, respectively, according to whether they were pathologically at a high risk.

Major surgical complications including ureteral injury and obstruction with a need for a double-J tube insertion, bladder fistula and intestinal obstruction were observed in five patients, with a complication rate of $6.8 \%(5 / 74)$. No treatment-related deaths were reported.

\section{Factors associated with the accuracy of pre- and postoperative accordance of PMI}

As shown in Figure 1, the use of imaging technologies including magnetic resonance imaging (MRI)/positron emission tomography-computed tomography (PET-CT) has largely increased after 2010 compared to that prior to 2010 . However, further analysis by Fisher's exact test showed that
Table I Patient characteristics $(\mathrm{N}=74)$

Cases n, (\%)

\begin{tabular}{|c|c|}
\hline \multicolumn{2}{|l|}{ Age (years) } \\
\hline$<40$ & $7(9.5)$ \\
\hline$\geq 40$ & $67(90.5)$ \\
\hline \multicolumn{2}{|l|}{ Pretreatment evaluation methods ${ }^{a}$} \\
\hline MRI & $26(35.1)$ \\
\hline PET-CT & $19(25.7)$ \\
\hline Pelvic examination under anesthesia & $14(18.9)$ \\
\hline \multicolumn{2}{|l|}{ Neoadjuvant treatment } \\
\hline None & $19(25.7)$ \\
\hline Neoadjuvant chemotherapy & $42(56.8)$ \\
\hline Neoadjuvant radio/chemoradiaotherapy & $13(17.6)$ \\
\hline \multicolumn{2}{|l|}{ Tumor size $(\mathrm{cm})$} \\
\hline$\leq 4 \mathrm{~cm}$ & $4 \mid(55.4)$ \\
\hline$>4 \mathrm{~cm}$ & $33(44.6)$ \\
\hline \multicolumn{2}{|l|}{ Histological subtype } \\
\hline Squamous cell carcinoma & $65(87.8)$ \\
\hline Adenocarcinoma & $9(12.2)$ \\
\hline \multicolumn{2}{|l|}{ Pathological PMI } \\
\hline Positive & $21(28.4)$ \\
\hline Negative & $53(71.6)$ \\
\hline \multicolumn{2}{|l|}{ LVSI } \\
\hline Positive & $27(36.5)$ \\
\hline Negative & $47(63.5)$ \\
\hline \multicolumn{2}{|l|}{ Pelvic lymph node metastasis } \\
\hline Positive & $3 I(4 I .9)$ \\
\hline Negative & $43(58.1)$ \\
\hline \multicolumn{2}{|l|}{ Deep stromal infiltration } \\
\hline Positive & $62(83.8)$ \\
\hline Negative & $12(16.2)$ \\
\hline \multicolumn{2}{|l|}{ Surgical margins } \\
\hline Positive & $7(9.5)$ \\
\hline Negative & $67(90.5)$ \\
\hline \multicolumn{2}{|l|}{ Postoperative treatment } \\
\hline None & $7(9.5)$ \\
\hline Chemotherapy & $12(16.2)$ \\
\hline Radio/chemoradiotherapy & $55(74.3)$ \\
\hline
\end{tabular}

Notes: aAll patients were routinely evaluated by pelvic examination and ultrasound in our study. The evaluation methods listed here include other image modalities except the routinely used methods mentioned earlier.

Abbreviations: MRI, magnetic resonance imaging; PET-CT, positron emission tomography-computed tomography; PMI, parametrial involvement; LVSI, lymphovascular space invasion.

the accuracy of MRI/PET-CT in detecting pathological PMI was lower than that of pelvic examination under anesthesia $(P=0.048)$.

In addition, readily obtained preoperative clinical variables, including chief complaints, use of MRI/PET-CT imaging, implementation of pelvic examination under anesthesia, neoadjuvant treatment and tumor size, were further assessed using a logistic analysis to identify factors associated with the accuracy of pre- and postoperative accordance of PMI. Neoadjuvant treatment was the only risk factor affecting the pre- and postoperative accordance rate of PMI (OR: 3.283 [95\% CI: 1.363-7.908], $P=0.008)$. In other words, 

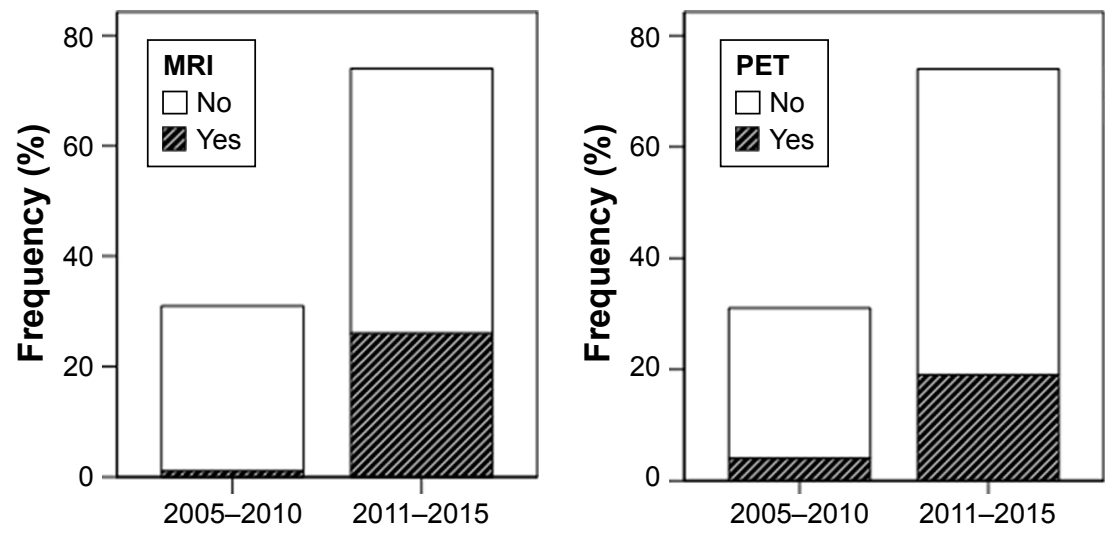

Figure I The usage of MRI and PET-CT in different periods of time.

Abbreviations: MRI, magnetic resonance imaging; PET-CT, positron emission tomography-computed tomography.

patients who received neoadjuvant treatment might have been downstaged and thus were eligible for subsequent radical surgery.

\section{Survival analysis}

The 2- and 5-year OS rates were $84.1 \%$ and $68.9 \%$, respectively, while the 2- and 5-year cumulative recurrence rates were $26.9 \%$ and $39.9 \%$, respectively (Figure 2). Multivariate proportional hazards modeling was carried out for OS and PFS analyses among subgroups according to age, admission time, pretreatment evaluation methods (MRI/ PET-CT/pelvic examination under anesthesia), neoadjuvant treatment, tumor size, histological type, deep stromal invasion, pelvic lymph node status, common iliac lymph node status, lymphovascular space invasion (LVSI), surgical margin, pre- and postoperative accordance of PMI, ovary preservation, operation time, blood loss, major surgical complications and postoperative treatment. The Cox model finally identified pre- and postoperative accordance of PMI, common iliac lymph node metastasis and major surgical
Table 2 Prognostic factors for OS and PFS selected by Cox's multivariate proportional hazard model

\begin{tabular}{|c|c|c|c|}
\hline & HR & $95 \% \mathrm{Cl}$ & $P$-value \\
\hline \multicolumn{4}{|l|}{ OS } \\
\hline Common iliac LN metastasis & 2.984 & I.147-7.766 & 0.025 \\
\hline $\begin{array}{l}\text { Pre- and postoperative } \\
\text { accordance of PMI }\end{array}$ & 2.889 & $1.158-7.206$ & 0.023 \\
\hline Major surgical complications & 3.243 & $1.115-9.433$ & 0.031 \\
\hline \multicolumn{4}{|l|}{ PFS } \\
\hline Common iliac LN metastasis & 2.827 & I.099-7.27| & 0.031 \\
\hline $\begin{array}{l}\text { Pre- and postoperative } \\
\text { accordance of PMI }\end{array}$ & $2.34 I$ & $0.894-6.127$ & 0.083 \\
\hline Major surgical complications & 2.875 & $0.986-8.383$ & 0.053 \\
\hline
\end{tabular}

Abbreviations: OS, overall survival; PFS, progression-free survival; LN, lymph node; PMI, parametrial involvement.

complications as significant prognostic factors for both OS and PFS (Table 2).

Nomograms for OS and PFS were then designed to visualize the Cox proportional hazards regression models. The nomograms for OS and PFS had bootstrap-corrected $\mathrm{C}$-indexes of 0.763 and 0.745 , respectively (Figure 3 ).
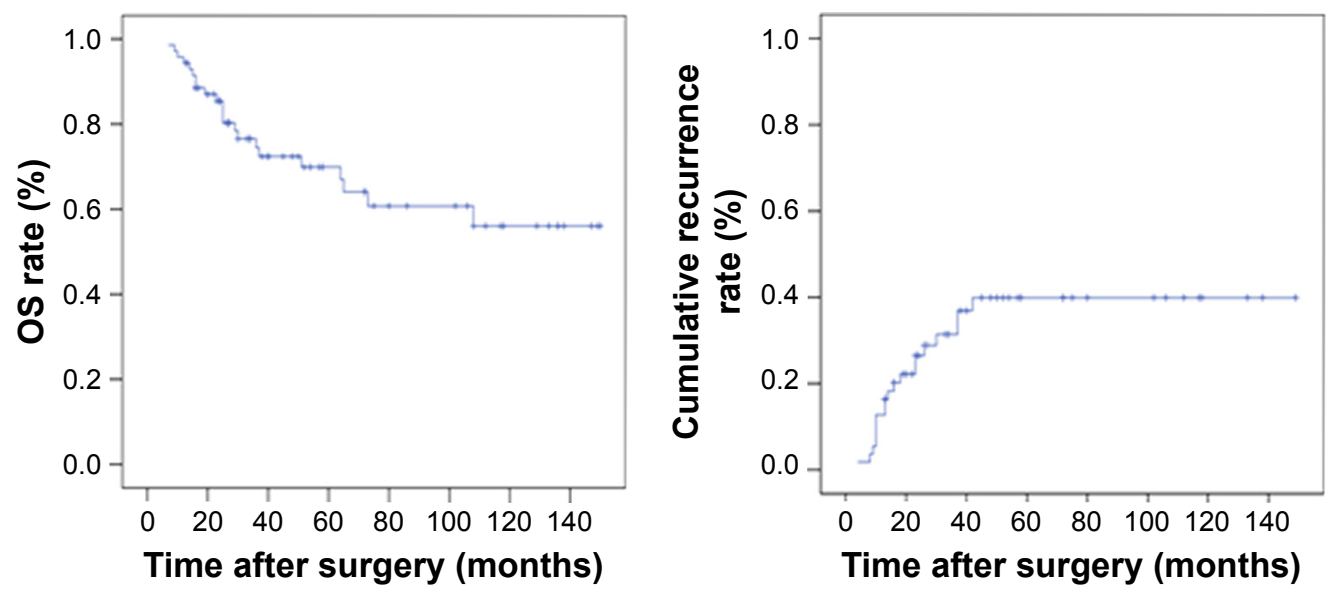

Figure 2 OS rate and cumulative recurrence rate of FIGO stage IIB patients. Abbreviation: OS, overall survival. 


PFS:
Points
Pre-and postoperative
accordance of PMI
Major surgical complications
Common iliac LN metastasis
Total points
1-year survival
2-year survival
3-year survival
5-year survival

OS:

Points

Pre- and postoperative accordance of PMI

Major surgical complications

Common iliac LN metastasis

Total points

1-year survival

2-year survival

3-year survival

5-year survival

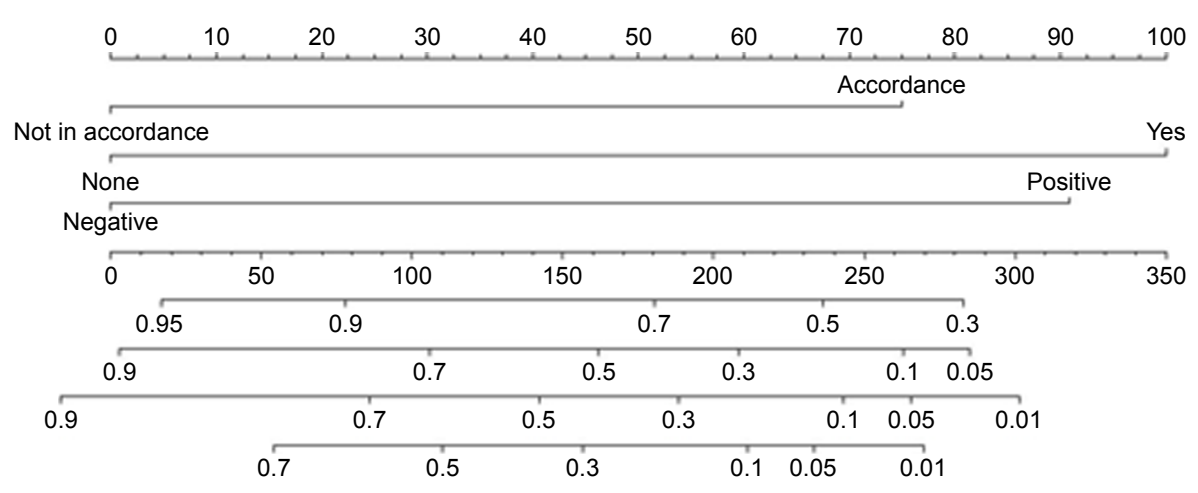

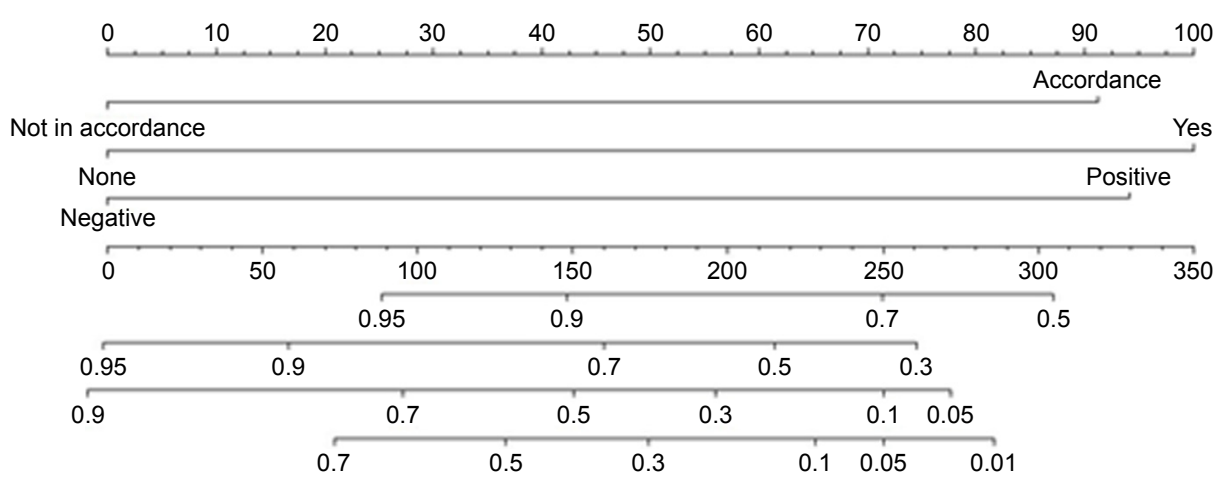

Figure 3 Nomograms for predicting PFS and OS, which had a bootstrap-corrected C-index of 0.763 and 0.745 , respectively.

Notes: To use, find patient's status of pre- and postoperative accordance of PMI, then draw straight line upward to points axis to determine how many points patient receives for accordance rate. Do this again for other axes, each time drawing straight line upward toward points axis. Sum points received for each predictor, and find sum on axis of total points. Draw straight line down to survival-probability axis to find patient's probability of I, 2, 3, 5-year PFS and OS.

Abbreviations: PFS, progression-free survival; OS, overall survival; C-index, concordance index; PMI, parametrial involvement; LN, lymph node.

\section{Discussion}

Cervical cancer is the second most common gynecological malignancy, with $>35 \%$ of tumors diagnosed at a locally advanced stage. ${ }^{16}$ Although radical surgery is generally accepted as a standard of care for patients with early stage disease and concurrent chemoradiotherapy for patients with advanced-stage disease, ${ }^{17}$ substantial controversies exist regarding the appropriate initial treatment approach for patients with LACC (FIGO stages IB2-IIB).

One of the reasons for the current lack of best treatment option for patients with FIGO stage IIB cervical cancer may be due to the difficulty in evaluating the "true" PMI before treatment. As the FIGO staging system is a clinical system, preoperatively discriminating the true pathological extent of the tumor from inflammatory changes, adhesion, fibrosis, and irregularly shaped, large-sized cervical tumors is difficult. ${ }^{8}$ In our study, although FIGO staging was assessed by at least two experienced gynecologists, and a consensus was reached, pathologically proven PMI was observed in only $47.3 \%$ of patients without neoadjuvant treatment and $28.4 \%$ of all patients. Similarly, in previous studies, the reported incidence of pathological PMI among patients with FIGO stage IIB disease ranged between $21.5 \%$ and $55 \% .{ }^{18}$ The low pre- and postoperative accordance of PMI implies that approximately one-half to three-fourths of patients clinically have "pseudo" stage IIB disease, which can be treated by radical surgery but not definitive radiation. In addition, the advantages of surgery include avoiding the long-term complications of radiation therapy and the morbidity of concurrent chemoradiation in patients who do not have high-risk pathologic factors, ie, positive nodes or parametrial and surgical margin involvement. In young patients, ovarian function and vaginal pliability can also be preserved. On the other hand, in cases with lymph node metastases, the ovaries may be transposed outside the radiation field for function preservation. ${ }^{18}$ With all these findings in the clinical context, treatment options for patients with FIGO stage IIB disease should be cautiously considered.

As the clinical assessment of PMI is important for making treatment plans, in the present study, we further compared the accuracy of different strategies in the preoperative detection of PMI. The long time span of this observational study was accompanied by a tendency of increasing use of imaging 
technologies including MRI/PET-CT after 2010. However, the specificity and sensitivity of MRI/PET-CT in assessing PMI were not as satisfactory as expected. Our findings revealed that the accuracy of MRI/PET-CT in detecting pathological PMI was lower than that of pelvic examination under anesthesia $(P=0.048)$. Yoon et $\mathrm{al}^{19}$ once compared the outcomes of MRI-based stage IIB cervical cancer with FIGO stage IIB cervical cancer and observed pathological PMI in only $32.6 \%$ of patients in the MRI group, and thus, the authors finally suggested that patients with MRI-based stage IIB disease with negative PMI on physical examination should be surgically treated because of a better survival rate than patients with FIGO stage IIB cervical cancer. Therefore, pelvic examination under anesthesia by experienced gynecologists should always be used as a cornerstone in the clinical evaluation of cervical cancer patients.

Currently, there are conflicting results in the literature regarding the use of neoadjuvant chemotherapy in treating LACC. A Phase III trial by Katsumata et $\mathrm{al}^{20}$ suggested that the benefit of neoadjuvant chemotherapy was to reduce the number of patients who received postoperative radiotherapy rather than improve OS. Other studies have indicated that neoadjuvant chemotherapy is a strong prognostic factor for PFS and OS. ${ }^{21,22}$ In the present study, neoadjuvant treatment was the only factor associated with the pre- and postoperative accordance of PMI, suggesting that patients receiving neoadjuvant treatment might be pathologically downstaged and thus be more suitable for subsequent radical surgery. Consequently, according to the data from our study and others, ${ }^{23,24}$ the benefit of neoadjuvant treatment may largely lie in reducing the tumor burden, achieving radical operability and reducing postoperative radiotherapeutic adverse effects.

In our study, the 5-year OS and cumulative recurrence rates were $68.9 \%$ and $39.9 \%$, respectively, which were comparable to those in patients treated with definitive radiotherapy (5-year OS ranging from 64\% to 69\%). ${ }^{25}$ In addition, the surgical complication rate was as low as $6.8 \%$ in our study, which was not higher than the average rate in early stage cervical cancer. ${ }^{26}$ Given that the prognosis of surgery and definitive radiotherapy may be approximately equivalent, the choice of treatment options for these patients can be largely individualized based on patient and tumor characteristics in the clinical context.

Prognostic factors for surgically treated patients with FIGO stage IIB disease were also analyzed in our study, and we found that the pre- and postoperative accordance of PMI, common iliac lymph node metastasis and major surgical complications were significant prognostic factors for both OS and PFS. Partially consistent with the Sedlis criteria, these factors might be more precise in discriminating highrisk patients who need further intensive treatment including radio/chemoradiotherapy. Furthermore, the identified risk factor of pre- and postoperative accordance of PMI demonstrates once again the importance of pretreatment clinical assessment of PMI, which awaits in-depth investigations for improving sensitivity and specificity.

Some degree of caution is necessary while interpreting the results of our study. Understandably, given the limitations of a retrospective study, potential confounding biases, such as selection bias introduced by surgeons in determining which patients should be considered for radical surgery plus adjuvant radiotherapy, may have been missed in the analysis. In addition, as in a specialized obstetrics and gynecology (OB/ GYN) hospital without radiotherapy facilities, the comparison of surgery and definitive chemoradiation could only be made using our data and data from the literature but not two groups of patients treated in the same hospital. In addition, this observational study lasted for $>10$ years, and the heterogeneity of the data, such as differences in the choice of neoadjuvant treatment, should be cautiously considered when interpreting the results of our analyses. However, our current findings provide evidence that the survival rate following radical surgery is comparable to that after generally accepted radical radiotherapy and that the surgical complication rate is relatively low. Therefore, radical hysterectomy might be a feasible alternative for patients with FIGO stage IIB cervical cancer. Considering the high percentage of "pseudo" PMI among patients with FIGO stage IIB disease, limited access to radiotherapy in China and a desire for hormone preservation among an increasing number of young patients, patients with FIGO stage IIB cervical cancer should be given individualized treatment in China. Furthermore, strategies to appropriately select patients who will benefit from surgery via pretreatment evaluation need to be further investigated.

\section{Acknowledgments}

This study was supported in part by National Natural Science Foundation of China (81571401 to L Yao and 81601235 to L Yuan) and by clinical technology innovation projects of Shanghai Shenkang Hospital Development Center (SHDC12016240 to L Yao). The abstract of this paper was presented at the 20th International Meeting of the European Society of Gynecological Oncology (ESGO 2017, November 4-7, 2017) as an e-poster with interim findings.

\section{Disclosure}

All authors declare that there are no conflicts of interests. 


\section{References}

1. Wiebe E, Denny L, Thomas G. Cancer of the cervix uteri. Int J Gynecol Obstet. 2012;119(2):S100-S109.

2. Jemal A, Bray F, Center MM, et al. Global cancer statistics. CA Cancer J Clin. 2011;61(2):69-90.

3. International Agency for Research on Cancer, World Health Organization. Cervical Cancer: Estimated Incidence, Mortality and Prevalence Worldwide in 2012. Lyon, Geneva: International Agency for Research on Cancer, World Health Organization; 2012.

4. Ying Q, Xia QM, Zheng RS. An analysis of incidence and mortality of cervical cancer in China, 2009. China Cancer. 2013;8:612-617.

5. Landoni F, Maneo A, Colombo A, et al. Randomised study of radical surgery versus radiotherapy for stage Ib-IIa cervical cancer. Lancet. 1997;350(9077):535-540.

6. Peters WA, Liu PY, Barrett RJ, et al. Concurrent chemotherapy and pelvic radiation therapy compared with pelvic radiation therapy alone as adjuvant therapy after radical surgery in high-risk early-stage cancer of the cervix. J Clin Oncol. 2000;18(8):1606-1613.

7. Eifel PJ, Winter K, Morris M, et al. Pelvic irradiation with concurrent chemotherapy versus pelvic and para-aortic irradiation for high-risk cervical cancer: an update of radiation therapy oncology group trial (RTOG) 90-01. J Clin Oncol. 2004;22(5):872-880.

8. Kasamatsu T, Onda T, Sawada M, Kato T, Ikeda S. Radical hysterectomy for FIGO stage IIB cervical cancer: clinicopathological characteristics and prognostic evaluation. Gynecol Oncol. 2009;114(1): 69-74.

9. Kawagoe T, Kashimura M, Matsuura Y, Sugihara K, Toki N, Aoki T. Clinical significance of tumor size in stage IB and II carcinoma of the uterine cervix. Int J Gynecol Cancer. 1999;9(5):421-426.

10. Carlson JA, Rusthoven C, Dewitt PE, Davidson SA, Schefter TE, Fisher CM. Are we appropriately selecting therapy for patients with cervical cancer? Longitudinal patterns-of-care analysis for stage IB-IIB cervical cancer. Int J Radiat Oncol Biol Phys. 2014;90(4):786-793.

11. Hasenburg A, Salama JK, van TJ, Amosson C, Chiu JK, Kieback DG. Evaluation of patients after extraperitoneal lymph node dissection and subsequent radiotherapy for cervical cancer. Gynecol Oncol. 2002; 84(2):321-326.

12. del Pino M, Fusté P, Pahisa J, et al. Laparoscopic lymphadenectomy in advanced cervical cancer: prognostic and therapeutic value. Int $J$ Gynecol Cancer. 2013;23(9):1675-1683.

13. Köhler C, Mustea A, Marnitz S, et al. Perioperative morbidity and rate of upstaging after laparoscopic staging for patients with locally advanced cervical cancer: results of a prospective randomized trial. Am J Obstet Gynecol. 2015;213(4):503.e1-50503.e1.

14. Robin TP, Amini A, Schefter TE, Behbakht K, Fisher CM. Disparities in standard of care treatment and associated survival decrement in patients with locally advanced cervical cancer. Gynecol Oncol. 2016; 143(2):319-325
15. Harrell FE [webpage on the Internet]. Rms: Regression Modeling Strategies. R Package Version 3.4-0. Available from: http://CRAN.R-project. org/package=rms. Accessed March 15, 2018.

16. Minig L, Patrono MG, Romero N, Rodríguez Moreno JF, Garcia-Donas J. Different strategies of treatment for uterine cervical carcinoma stage IB2-IIB. World J Clin Oncol. 2014;5(2):86-92.

17. Quinn MA, Benedet JL, Odicino F, et al. Carcinoma of the cervix uteri. FIGO 26th Annual Report on the Results of Treatment in Gynecological Cancer. Int J Gynaecol Obstet. 2006;95(Suppl 1):S43-S103.

18. Suprasert P, Srisomboon J, Kasamatsu T. Radical hysterectomy for stage IIB cervical cancer: a review. Int J Gynecol Cancer. 2005;15(6): 995-1001.

19. Yoon A, Park JJ, Park BK, et al. Long-term outcomes of MRI stage IIB cervical cancer. Int J Gynecol Cancer. 2016;26(7):1252-1257.

20. Katsumata N, Yoshikawa H, Kobayashi H, et al. Phase III randomised controlled trial of neoadjuvant chemotherapy plus radical surgery vs radical surgery alone for stages IB2, IIA2, and IIB cervical cancer: a Japan Clinical Oncology Group trial (JCOG 0102). Br J Cancer. 2013; 108(10):1957-1963.

21. Landoni F, Sartori E, Maggino T, et al. Is there a role for postoperative treatment in patients with stage Ib2-IIb cervical cancer treated with neo-adjuvant chemotherapy and radical surgery? An Italian multicenter retrospective study. Gynecol Oncol. 2014;132(3):611-617.

22. Ye Q, Yuan HX, Chen HL. Responsiveness of neoadjuvant chemotherapy before surgery predicts favorable prognosis for cervical cancer patients: a meta-analysis. J Cancer Res Clin Oncol. 2013;139(11): 1887-1898.

23. Dueñas-Gonzalez A, López-Graniel C, González-Enciso A, et al. A phase II study of multimodality treatment for locally advanced cervical cancer: neoadjuvant carboplatin and paclitaxel followed by radical hysterectomy and adjuvant cisplatin chemoradiation. Ann Oncol. 2003;14(8):1278-1284.

24. Gong L, Zhang JW, Yin RT, et al. Safety and efficacy of neoadjuvant chemotherapy followed by radical surgery versus radical surgery alone in locally advanced cervical cancer patients. Int J Gynecol Cancer. 2016; 26(4):722-728.

25. Mabuchi S, Okazawa M, Isohashi F, et al. Radical hysterectomy with adjuvant radiotherapy versus definitive radiotherapy alone for FIGO stage IIB cervical cancer. Gynecol Oncol. 2011;123(2):241-247.

26. Gallotta V, Conte C, Federico A, et al. Robotic versus laparoscopic radical hysterectomy in early cervical cancer: A case matched control study. Eur J Surg Oncol. 2018;44(6):754-759.
OncoTargets and Therapy

\section{Publish your work in this journal}

OncoTargets and Therapy is an international, peer-reviewed, open access journal focusing on the pathological basis of all cancers, potential targets for therapy and treatment protocols employed to improve the management of cancer patients. The journal also focuses on the impact of management programs and new therapeutic agents and protocols on

\section{Dovepress}

patient perspectives such as quality of life, adherence and satisfaction. The manuscript management system is completely online and includes a very quick and fair peer-review system, which is all easy to use. Visit http://www.dovepress.com/testimonials.php to read real quotes from published authors. 